
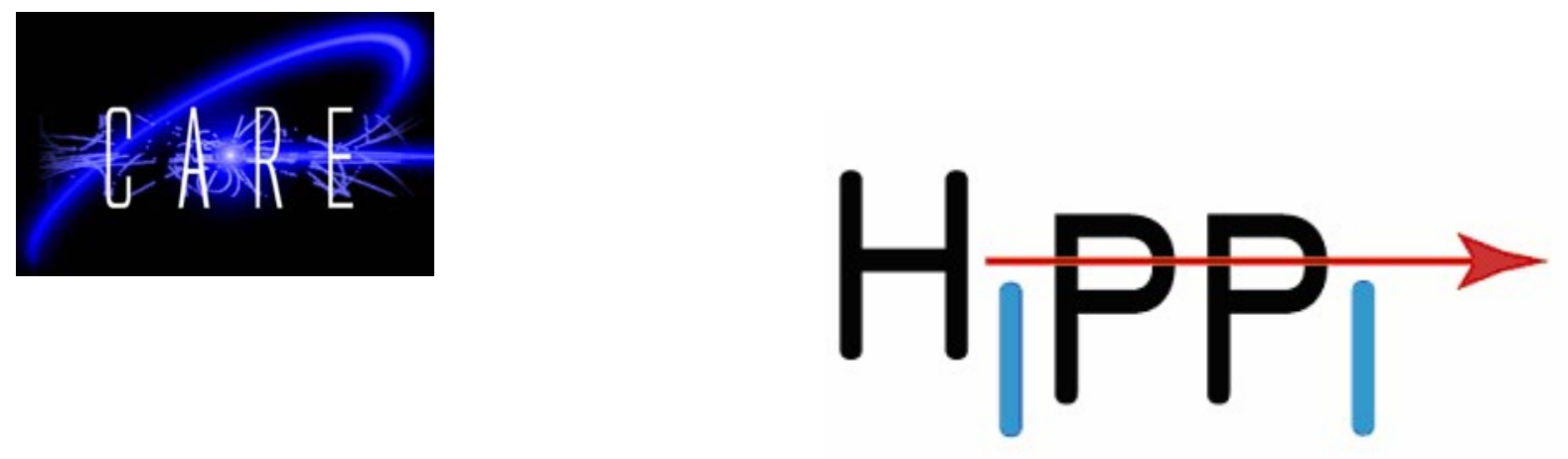

\title{
IMPROVEMENT OF THE BLADE TUNER DESIGN FOR SUPERCONDUCTING RF CAVITIES
}

\author{
Carlo Pagani, Angelo Bosotti, Paolo Michelato, Nicola Panzeri, Paolo Pierini \\ INFN Milano - LASA, Italy
}

\begin{abstract}
As of today, no complete technological solution exists for a cold tuning system fulfilling the requirements envisaged for the International Linear Collider, based on the superconducting RF technology. We present here the design improvements for the blade tuner, a coaxial device, which can provide both the slow structure tuning and the fast tuning capabilities needed for Lorentz Force Detuning (LFD) compensation and microphonics stabilization (by means of the integration with a piezoelectric system). The system has been originally built by INFN and installed and tested at DESY on the superstructures, since it is located around the cavity helium vessel and does not require longitudinal clearance. Its design here is parametrically reviewed in terms of the requirements for higher accelerating fields and of the mechanical integration with a piezoelectric based system for the LFD and microphonics compensation.
\end{abstract}

Contribution to the PAC05, Knoxville, USA

Work supported by the European Community-Research Infrastructure Activity under the FP6 "Structuring the European Research Area" programme (CARE, contract number RII3-CT2003-506395). 


\title{
IMPROVEMENT OF THE BLADE TUNER DESIGN FOR SUPERCONDUCTING RF CAVITIES
}

\author{
Carlo Pagani, Angelo Bosotti, Paolo Michelato, Nicola Panzeri, Paolo Pierini \\ INFN Milano - LASA, Italy
}

\begin{abstract}
As of today, no complete technological solution exists for a cold tuning system fulfilling the requirements envisaged for the International Linear Collider, based on the superconducting RF technology. We present here the design improvements for the blade tuner, a coaxial device, which can provide both the slow structure tuning and the fast tuning capabilities needed for Lorentz Force Detuning (LFD) compensation and microphonics stabilization (by means of the integration with a piezoelectric system). The system has been originally built by INFN and installed and tested at DESY on the superstructures, since it is located around the cavity helium vessel and does not require longitudinal clearance. Its design here is parametrically reviewed in terms of the requirements for higher accelerating fields and of the mechanical integration with a piezoelectric based system for the LFD and microphonics compensation.
\end{abstract}

\section{THE TTF SUPERSTRUCTURES TUNER}

The design of a coaxial tuner solution for superconducting structures was originally motivated from the need of a cold tuner for the TTF superstructures tests [1]. For this case the standard TTF tuner solution, with its longitudinal clearance, would have been incompatible with the foreseen increase in filling factor. The coaxial blade tuner [2], inspired by the idea described in Ref. [3], was then proposed both for the reduced cavity spacing foreseen by the TESLA TDR and for the superstructures, and prototypes were built and tested in the CHECHIA vertical cryostat and on the TTF linac in 2002 [1]. During both tests the blade tuner performed as expected in terms of stiffness, frequency sensitivity and tuning capability. Figure 1 shows one 2-cavity superstructure equipped with blade tuners during assembly into a TTF module.

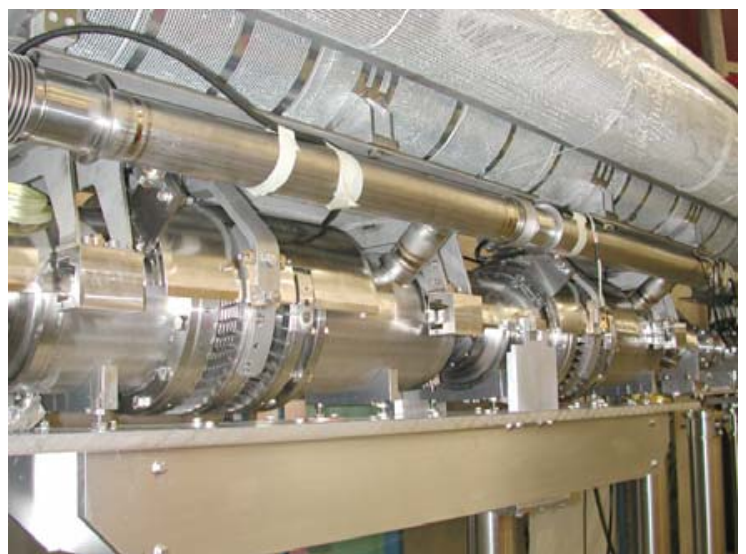

Figure 1: One 2-cavity superstructure, equipped with blade tuners, during assembly in TTF.

\section{EVOLUTION OF THE TUNER DESIGN}

The tuner design presented in Ref. [2] and tested on the TTF linac provided the necessary slow tuning action, but did not demonstrate the fast tuning action needed both for the high gradients $(\geq 30 \mathrm{MV} / \mathrm{m})$ at which the International Linear Collider will operate and for the active compensation of microphonics in CW operation, for ERL and FEL applications. We have therefore updated the tuner design in order to include the possibility of a fast tuning action, by means of the integration of an active device of the piezoelectric or magnetostrictive type.

\section{Tuner design}

The main tuner mechanism consists of a three-ring bending system connected by welded blades at an angle which transforms the azymuthal rotation (in opposite directions) of the two halves of the central ring into a variation of the distance between the end rings. The azymuthal rotation is provided by a leverage system connected to a step motor. The leverage system amplifies the torque of the step motor, contemporarily increasing the tuning sensitivity.

In the standard design (reported in [2]) the two end rings are rigidly connected to the cavity He tank, which has been split in two halves joined by a bellow in order to allow the variation of the cavity length.

The bending system has been designed with a parametrical model, in terms of the blade length and inclination to define the structure kinematics and in terms of number and position of blades to provide the axial stiffness and tuning force compatibility.

\section{Integration of the LFD compensation system}

The parametrical model has been then verified by means of finite element models, which provided more accurate information; such as stresses, deformations and stiffness not only in the axial but also in the transversal and torsional directions. Thess detailed informations have then been used for the integration of a fast tuning action based on piezoelectric actuators, which are subject to several requirements limiting the choice of possible design configurations.

First of all, the piezoelectric actuators (piezos) cannot be subject to tension forces, and bending and shear actions need to be carefully avoided. Moreover, in order to maximize their lifetime, a correct preload range needs to be guaranteed under all operating condition. In addition to that, the characteristic behavior of piezo operation at cryogenic temperatures, in terms of stroke and preload effects, is still a subject undergoing a systematic investigation, especially within the CARE program [4]. 
From all the considerations expressed above, and using as guidelines the typical values of the TESLA type structures in terms of cavity elasticity and tuning requirements (see Table 1), we have integrated the piezoelectric elements in the tuner design, by inserting two elements between one of the end rings and the corresponding flange on the $\mathrm{He}$ vessel, as shown in Figure 2.

Table 1: Guidelines for a TESLA cavity tuner.

\begin{tabular}{|l|c|c|}
\hline & $\begin{array}{c}\text { Frequency range } \\
{[\mathrm{kHz}]}\end{array}$ & $\begin{array}{c}\text { Axial movement } \\
{[\mu \mathrm{m}]}\end{array}$ \\
\hline Slow tuning & $\sim 500$ & $\sim 1500$ \\
\hline Fast tuning & $\sim 1$ & $\sim 3$ \\
\hline
\end{tabular}

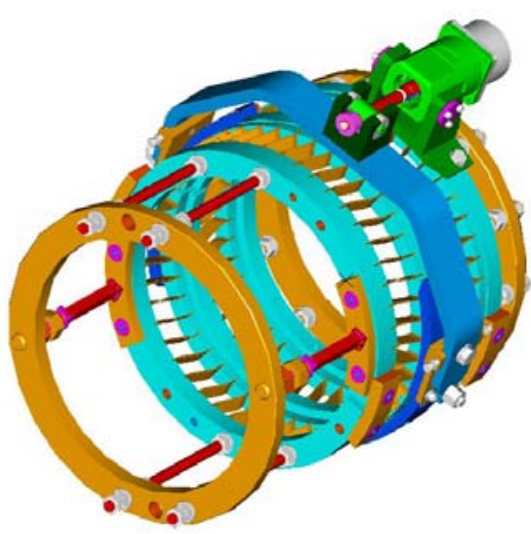

Figure 2. The piezo-assisted tuner.

Under this configuration, the cavity elasticity is used to provide a controlled preload to the piezoelectric elements, and thus the cavity has to be tuned to operate in a stretched condition and the working points are obviously chosen in order to avoid an inversion of the direction of the internal stress in the blades and piezos over the complete tuning ranges.

The current piezo capabilities assessed up to now [4] seems to satisfy the operating requirements; furthermore, different piezo with different cross sections, or even magnetostrictive elements, can be accommodated in the prototype under construction with minimal variation. Thus the final choice of piezo actuators can be safely postponed after their complete characterization in terms of precise values of stroke and necessary preload conditions.

\section{Characterization of the full system}

The axial mechanical behavior of the full system (cavity + He tank + tuner) has been investigated parametrically by means of the spring model shown in Fig. 3. The stiffness of every part, for the TESLA cavity case, is reported in Table 2, together with the material types, the elastic properties (Young module) and the legend of symbols used in Fig. 3. The stiffness values were either derived from FEM analysis or measured experimentally.
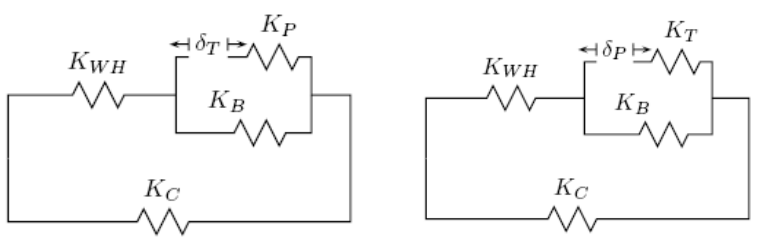

Figure 3: Equivalent spring models

Table 2: Characteristics used in the spring model.

\begin{tabular}{|c|c|c|c|c|}
\hline Part & Material & $\begin{array}{c}\text { Stiffness } \\
\text { symbol }\end{array}$ & $\mathrm{E}(\mathrm{MPa})$ & $\begin{array}{c}\mathrm{K} \\
(\mathrm{N} / \mu \mathrm{m})\end{array}$ \\
\hline He tank & $\mathrm{Ti}$ & $K_{H}$ & 119000 & 440 \\
\hline Tuner & $\mathrm{Ti}$ & $K_{T}$ & 119000 & 25 \\
\hline Cavity & $\mathrm{Nb}$ & $K_{C}$ & 105000 & 3.213 \\
\hline End disk & $\mathrm{Nb}$ & $K_{W}$ & 105000 & 27.1 \\
\hline Piezo & --- & $K_{P}$ & --- & $2 \times 105$ \\
\hline $\begin{array}{c}\text { Tank } \\
\text { bellow }\end{array}$ & $\mathrm{Ti}$ & $K_{B}$ & 119000 & 0.228 \\
\hline
\end{tabular}

The forces and displacements of every part in the system corresponding to a tuner displacement $\left(\delta_{\mathrm{T}}\right)$ of $1 \mathrm{~mm}$ (slow tuning action) or a piezo displacement $\left(\delta_{\mathrm{p}}\right)$ of $1 \mu \mathrm{m}$ (fast tuning action) have been evaluated and are reported in Table 3.

We note here that due to the low stiffness of the end disks, only $87 \%$ of the displacement applied by the tuner is transferred to the cavity. For the fast tuning action a further contribution comes from the tuner stiffness, and $79 \%$ of the displacement applied by the piezos is transferred to the cavity. In particular, to provide the values reported in Table 1 the piezo has to assure a maximum stroke of $\sim 4 \mu \mathrm{m}$ at $2 \mathrm{~K}$. The use of stiffer end disks for the He tank connection would slightly reduce the requirements on tuner/piezo excursion.

It is also important to point out that the piezo preload is varying with the slow tuner action, therefore a suitable pre-tuning strategy needs to be assessed to ensure that during the slow tuning action the preload remains within valid operation limits. Furthermore, we have to ensure that the piezo preload has to be always lower than its blocking force (the force above which the piezo do not provide stroke). For the prototype case of two piezos, with a cross section of $10 \times 10 \mathrm{~mm}$, a blocking force of 4 $\mathrm{kN}$ (each) is expected and a maximum slow tuning action of $\sim 800 \mathrm{kHz}$ can be safely applied, which is at least a factor of two higher than the current warm-cold frequency reproducibility at the TTF. The experimental tests planned in the context of the CARE program to assess the thermal expansion of different piezo-ceramic materials together with the cold tests of the tuner prototypes (see below for details) will further reduce the overall uncertainty of the preload at the tuner working point. Moreover, the needed cold tuning action is expected to further reduce for the case of a big industrial cavity production, like the one needed by the XFEL or ILC cases. 
Table 3: Axial forces and displacements for a tuner elongation of $1 \mathrm{~mm}$ (slow tuning action) and for a piezo elongation of $1 \mu \mathrm{m}$ (fast tuning action).

\begin{tabular}{|c|c|c|}
\hline \multicolumn{3}{|c|}{ Load case: $\delta_{\mathrm{T}}=1 \mathrm{~mm}$ (slow tuning) } \\
\hline Part & Axial force $(\mathrm{N})$ & Axial displ. $(\mathrm{mm})$ \\
\hline He + disk & -2812.5 & -0.110 \\
\hline Tuner & -3037.0 & 1.000 \\
\hline Cavity & 2812.5 & 0.876 \\
\hline Piezo & $-3037.0 / 2$ & -0.014 \\
\hline He bellow & 224.5 & 0.986 \\
\hline \multicolumn{3}{|c|}{ Load case $\delta_{\mathrm{P}}=1 \mu \mathrm{m}$ (fast tuning) } \\
\hline Part & Axial force $(\mathrm{N})$ & Axial displ. $(\mu \mathrm{m})$ \\
\hline He + disk & -2.540 & -0.100 \\
\hline Tuner & -2.743 & -0.110 \\
\hline Cavity & 2.541 & 0.790 \\
\hline Piezo & -2.743 & 1.000 \\
\hline He bellow & 0.203 & 0.890 \\
\hline
\end{tabular}

\section{Adaptation to the TTF CRY-3 design}

According to the design illustrated in the preceding sections, we have started the fabrication of two complete prototypes for the tuning system (including the leverage system, split He Tank and ancillary components). The system is designed to be fully compatible with the actual TTF cryomodule design (CRY-3), which is the base for the future developments needed for the industrial ILC and XFEL modules.

Both tuner prototypes will be delivered in mid-2005, and we plan tests of the combined slow/fast tuning capabilities in CHECHIA. Their final installation in the high gradient TTF Module 6 is still open.

\section{Adaptation to low beta elliptical structures}

In the context of the HIPPI subproject of the CARE program [5], our group is going to equip the two low beta (0.47) $700 \mathrm{MHz}$ elliptical cavities, developed for the TRASCO project, for high power pulsed measurements in CRYHOLAB at CEA/Saclay, to verify the feasibility of LFD compensation of these structures. The cavities have been tested in CW conditions up to $17 \mathrm{MV} / \mathrm{m}(61 \mathrm{MV} / \mathrm{m}$ peak electric and $100 \mathrm{mT}$ peak magnetic fields) [6]. The test of these structures under pulsed operation is particularly significant for the feasibility studies of superconducting high power proton drivers.

On the basis of the design procedures described in the preceding sections, a scaling of the coaxial tuner, and its adaptation to the TRASCO cavity case, has been performed, and the layout is shown in Fig. 4. The construction of the tuner prototypes for the HIPPI program is foreseen in late 2006, thus the final detailed engineering of the tuner will be finalized after the end of the tests that will be performed at DESY on the TESLA type tuner.

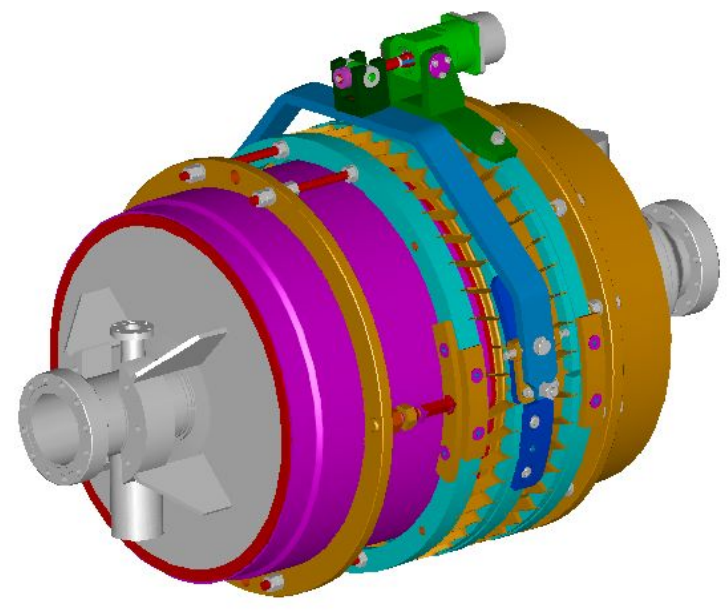

Figure 4: The preliminary layout of the scaled coaxial tuner for the $700 \mathrm{MHz} 0.5$ beta TRASCO cavity.

\section{CONCLUSIONS}

The tests of a complete blade tuner prototype integrating an active capability for LFD and microphonics compensation will be performed soon. We expect the tests will provide the final qualification of this solution, which is attractive in terms of compactness and longitudinal clearance needs.

\section{ACKNOWLEDGEMENTS}

We acknowledge the contribution of H.-B. Peters, R. Lange, and the DESY MKS group, in the design of the leverage system and the experimental characterization of the blade tuner. This work is partially supported by the European Community-Research Infrastructure Activity under the FP6 "Structuring the European Research Area" programme (CARE, contract number RII3-CT-2003506395).

\section{REFERENCES}

[1] J. Sekutowickz et al., "Test of Two $\mathrm{Nb}$ Superstructure Prototypes", in Phys. Rev. STAB, 7, 012002 (2004).

[2] D. Barni, A. Bosotti, C. Pagani, R. Lange, H.B. Peters, "A New Tuner for TESLA", in Proceedings of EPAC2002, Paris, France, p. 2205.

[3] H. Kaiser, "New Approaches to Tuning of TESLA Resonators", in Proceedings of SRF Workshop 1999, Santa Fe, USA.

[4] P. Sekalski et al., "Static Absolute Force Measurement for Preloaded Piezoelements used for Active Lorentz Force Detuning System", in Proceedings of Linac 2004, Lubeck, Germany, p. 486.

[5] See http://mgt-hippi.web.cern.ch/mgt-hippi/ and http://esgard.lal.in2p3.fr/ for an outline of the CARE program and the HIPPI activities.

[6] A. Bosotti et al. "RF Tests of the Beta $=0.5$ Five Cell TRASCO Cavities", in Proceedings of EPAC2004, Lucerne, Switzerland, p. 1024. 